УДК 004.942.001.57

\title{
ПОСТРОЕНИЕ АНИЗОТРОПНОЙ ГИДРОДИНАМИЧЕСКОЙ МОДЕЛИ И ИССЛЕДОВАНИЕ ВЛИЯНИЯ АНИЗОТРОПИИ ПРОНИЦАЕМОСТИ НА ПРИМЕРЕ МОДЕЛИРОВАНИЯ БЛОКА МЕСТОРОЖДЕНИЯ
}

\section{Ермеков Роман Игоревич',}

trommka@mail.ru

\author{
Коровин Михаил Олегович', \\ korovinmo@hw.tpu.ru
}

\author{
Меркулов Виталий Павлович', \\ merkulovvp@hw.tpu.ru
}

\author{
Чернова Оксана Сергеевна', \\ chernovaos@hw.tpu.ru \\ 1 Национальный исследовательский Томский политехнический университет, \\ Россия, 634050, г. Томск, пр. Ленина, 30.
}

Актуальность исследования определена тем, что наличие адаптированной под исторические данные эксплуатации геолого-гидродинамической модели месторождения является мощным инструментом, с помощью которого инженеры имеют возможность проводить мониторинг текущей ситуации и рассматривать эффективность предлагаемых решений в ближайшем будущем. Поэтому для получения достоверных результатов весьма важным является качество построения модели коллекторов с учётом их геологических особенностей. Одной из таких особенностей можно считать анизотропию проницаемости. Весьма важной она является при рассмотрении коллекторов с низкими фильтрационно-емкостными свойствами, к разработке которых на текущий момент активно начали приступать нефтегазодобывающие компании.

В цели данной работы входила оценка влияния анизотропии проницаемости на поведение гидродинамической модели продуктивного коллектора нефтяного месторождения.

Объектом исследования является одно из нефтяных месторождений Томской области, состоящее из терригенных отложений. Особенностью месторождения является его месторасположение в системе сложных локальных поднятий разного порядка. Основной продуктивный коллектор представлен юрскими отложениями, сформировавшимися в регрессивно-трансгрессивную серию осадконакопления и имеющими сложное распределение фильтрационно-емкостных свойств.

Методы исследования основаны на построении модели месторождения и проведении гидродинамических расчётов, включающих подбор близкого к реальности значения анизотропии проницаемости на основе промысловых данных, оптимизацию ранее существующей системы разработки, а также построение и анализ карт разработки.

В результате исследования было установлено, что упущение анизотропии проницаемости приводит к завышению накопленных показателей разработки месторождений. Было получено, что повышение величины анизотропии не всегда приводит к росту накопленной добычи нефти, что несомненно подчеркивает особенность геологического строения коллектора. На завершающем этапе был проведён гидродинамический расчёт разработки в срок на 15 лет, по результатам которого также сделаны выводы о правильности применения операций по повышению нефтеотдачи.

\section{Ключевые слова:}

Анизотропия проницаемости, масштаб анизотропии, направленность анизотропии, гидродинамическое моделирование, система разработки.

\section{Введение}

Общеизвестно что проницаемость является векторной величиной и её изменение происходит в трёх взаимно ортогональных плоскостях [1]. Как следствие, проницаемость можно представлять как тензор третьего порядка и рассматривать как прямоугольный параллелепипед:

$$
\bar{k}=\left[\begin{array}{lll}
k_{x x} & k_{x y} & k_{x z} \\
k_{y x} & k_{y y} & k_{y z} \\
k_{z x} & k_{z y} & k_{z z}
\end{array}\right],
$$

где $k_{x x}, k_{y y}, k_{z z}$ - элементы главной диагонали матрицы, являющиеся ортогональными к плоскостям параллелепипеда. Однако при таком представлении описание явления анизотропии проницаемости сильно усложняется и пока не имеет решения. Поэтому в общем случае принимается упрощенное представление, суть которого заключается в предположении вектора проницаемости как тензора второго порядка:

$$
\bar{k}=\left[\begin{array}{l}
k_{i i} k_{i j} \\
k_{j i} k_{j j}
\end{array}\right],
$$

где $i, j$ - индексы, характеризующие определенное направление вектора $(x, y, z)$. Допущением здесь является то, что касательные составляющие $k_{i j}=k_{i j}=1$ (рис. 1$)$.

Внесение описанного допущения является мерой вынужденной, но тем не менее уже позволяющей проводить исследование явления, которое подкреплено достаточно хорошей математической основой.

Некоторые авторы связывают явление анизотропии проницаемости с литолого-петрофизической неоднородностью пород коллекторов. Под ней 
в общем случае понимается существование согласованных изменений в структуре и текстуре горной породы, которые определенно ориентированы в пространстве. На данный момент хорошо известно, что создание таких согласованных изменений происходит по двум причинам: взаимодействие нескольких обстановок осадконакопления при формировании резервуара и влияние последующих постседиментационных процессов.

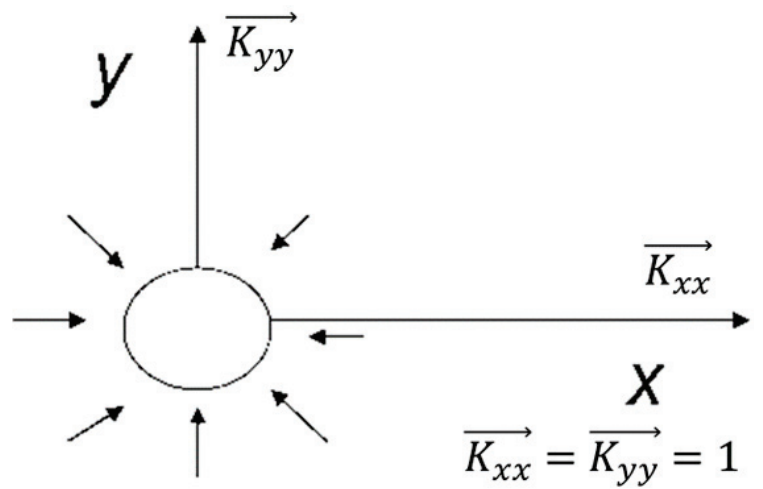

Рис.1. Упрощенное представление об анизотропии проницаемости [2.C.12]

Fig. 1. Simplified view of permeability anisotropy [2. P.12]

Формирование резервуара в различных обстановках осадконакопления имеет ключевое влияние на конечную структуру и текстуру слагающих его пород. Это связано как с типом материала осадка зёрен, так и с их размером и сортировкой. Последние имеют прямую зависимость от величины энергии, при которой происходит их перемещение от места их формирования до места осаждения. Известно, что основным транспортировщиком осадков является вода. Именно направление движения основного потока измельчает, дробит, сортирует и вытягивает зёрна в определенном направлении. Это приводит к тому, что направление осадка, которое соответствует направлению вытянутости зёрен имеет в конечном счёте улучшенные фильтрационные свойства, чем в ортогональном. При смене обстановки осадконакопления происходят серьезные изменения в литолого-петрофизическом состоянии. Однако такие процессы не являются мгновенными и происходят длительное время. После формирования резервуара его дальнейшее погружение может сопровождаться значительным проявлением вторичных процессов. Такими процессами могут являться тектонические движения, приводящие к деформации пород, процессы метасоматизма, цементация, карбонатизация и т. д. Все рассмотренные явления формируют конечные неоднородные фильтрационные характеристики горных пород, что и проявляется в явлении анизотропии проницаемости [3].

Таким образом, анизотропию проницаемости можно характеризовать как направленностью, так и масштабом проявления. Она является ещё одной важной геологической характеристикой резервуара, которую необходимо учитывать при постро- ении геологической модели для получения в дальнейшем более корректных результатов гидродинамических расчётов.

\section{Построение геологической}

и гидродинамической моделей

В данной работе все гидродинамические расчёты выполнялись в ПО Petrel Schlumberger [4]. Для построения модели пласта были использованы следующие входные данные:

1) координаты положения устьев двадцать одной скважин и их альтитуды;

2) инклинометрия скважин;

3) структурная карта подошвы Баженовской свиты, полученная по сейсмическим данным;

4) отбивки пласта $Ю_{1}^{3}$, выявленные по каждой скважине;

5) интерпретированные каротажные данные.

Процесс геологического моделирования состоит из четырех стадий:

1. Структурное моделирование и построение ячеистой модели.

2. Фациальное моделирование.

3. Петрофизическое моделирование.

4. Моделирование флюидонасыщения.

\section{Структурное моделирование}

Первым шагом при структурном моделировании являлся загрузка каждой скважины по заданным координатам и инклинометрии. Далее проводилось создание структурной карты поверхности, которая проецировалась по отбивкам пласта $Ю_{3}^{1}$. Сама структурная карта создавалась с помощью метода интерполяции и была обрезана по размеру рассматриваемого участка.

На втором шаге проводилось построение ячеистой модели. Размер ячеек по горизонтали был выбран $50 \times 50$ м ввиду малых размеров рассматриваемого участка. Размер ячеек по вертикали составил 0,5 м. Такая величина позволила не упустить малые пропластки при фациальном моделировании данных ГИС и не затрачивать много времени на проведение расчётов на более малой сетке. Сам размер был выбран посредством анализа диапазона значений от 0,2 до 0,7 м.

В заключение можно отметить, что размер созданной ячеистой модели составил $5 \times 5$ км по горизонтали и 21,8 м по вертикали. Количество ячеек составило 1040000.

\section{Фациальное моделирование}

Согласно исходным данным, интерпретированные каротажные данные содержали в себе разбиение на фации по гидродинамическим единицам потока [5]. Для построения фациальной модели был использован метод Truncated Gaussian simulation, применяемый как метод, хорошо характеризующий гетерогенные резервуары и обстановки, имеющие неравномерное чередование петрофизических свойств, что справедливо для описываемой обстановки прибрежных баровых песчаников [6]. 
Далее, согласно общим правилам, происходило создание куба фаций через определение параметров вариограмм. Сами параметры отражены в табл. 1 и были взяты как рекомендуемые [7]. Параметры вариограмм для моделирования самих фаций подбирались в соответствии с их процентом, представленным по всей мощности пласта (табл. 2). Фация восемь является фацией, представляющей неколлектор (рис. 2).

Таблица 1. Параметры вариограммы для фациального моделирования

Table 1. Variogram parameters for facies modeling

\begin{tabular}{|c|c|c|}
\hline $\begin{array}{c}\text { Пласт } \\
\text { Layer }\end{array}$ & $\begin{array}{c}\text { Тип вариограммы } \\
\text { Variogram type }\end{array}$ & $\begin{array}{c}\text { Kopреляционная длина, } \mathrm{m} \\
\text { Correlation length, } \mathrm{m}\end{array}$ \\
\hline \multirow{3}{*}{$\aleph_{1}^{3}$} & Главная (Major) & 18000 \\
\cline { 2 - 3 } & Второстепенная (Minor) & 17800 \\
\cline { 2 - 3 } & Вертикальная (Vertical) & 33,36 \\
\hline
\end{tabular}

Таблица 2. Параметры вариограмм для каждой фации

Table 2. Variogram parameters for each facies

\begin{tabular}{|c|c|c|c|c|}
\hline $\begin{array}{c}\text { Фация } \\
\text { Facies }\end{array}$ & $\begin{array}{l}\text { Процент от мощности, \% } \\
\text { Percentage of thickness, \% }\end{array}$ & $\begin{array}{c}\text { Мощность, } \\
\text { Thickness, } \mathrm{m}\end{array}$ & $\begin{array}{c}\text { Ранг, м } \\
\text { Range, } \mathrm{m}\end{array}$ & $\begin{array}{c}\text { Наггет, } \mathrm{M} \\
\text { Nugget, } \mathrm{m}\end{array}$ \\
\hline 1 & 2,34 & 0,498 & 0,998 & 0,0001 \\
\hline 2 & 1,7 & 0,362 & 0,714 & 0,0001 \\
\hline 3 & 4,84 & 1,029 & 1,265 & 0,0001 \\
\hline 4 & 6,26 & 1,332 & 1,363 & 0,0001 \\
\hline 5 & 12,51 & 2,661 & 3,302 & 0,2586 \\
\hline 6 & 6,96 & 1,480 & 2,081 & 0,2279 \\
\hline 7 & 23,68 & 5,036 & 6,432 & 0,3239 \\
\hline 8 & 41,71 & 8,872 & 12,388 & - \\
\hline
\end{tabular}

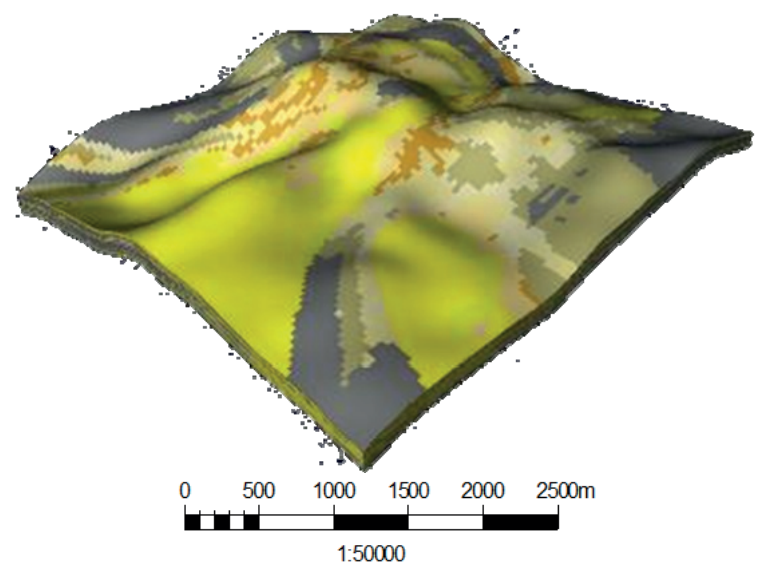

Symbol legend

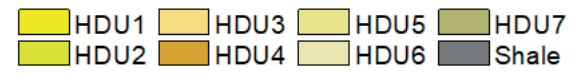

Рис. 2. Фациальная модель пласта $Ю_{1}{ }^{3}$

Fig. 2. Facies model of $\wp_{1}^{3}$ layer

Следующим шагом в расчётах стало рассмотрение влияния на поведение модели внедрения латеральной анизотропии проницаемости. Для этого куб проницаемости модели был умножен на коэффициент 1,48 в направлении оси X, в то время как к направлению $\mathrm{Y}$ был применен коэффициент 0,67 в соответствии с результатами расчётов ани- зотропии [8-11]. Итоги проведенного модельного расчёта в этом случае представлены в табл. 3 и на рис. 3,4 , где в табл. $3 a_{z}$ - величина вертикальной анизотропии (vertical anisotropy), $Q_{0}$ - накопленная добыча нефти (cumulative oil production), $Q_{w}-$ накопленная добыча воды (cumulative water production), $Q_{i n j}$ - накопленная закачка воды (cumulative water injection), КИН - коэффициент извлечения нефти (oil recovery factor).

Таблица 3. Результаты вариации вертикальной и латеральной анизотропий

Table 3. Results of vertical and lateral anisotropy variation

\begin{tabular}{|c|c|c|c|}
\hline \multicolumn{4}{|c|}{ Сценарий, млн м ${ }^{3} /$ Scenario, MM m³ } \\
\hline$a_{z}$ & $\begin{array}{c}\text { Пессимистичный } \\
\text { Pessimistic }\end{array}$ & $\begin{array}{c}\text { Наиболее вероятный } \\
\text { Most likely }\end{array}$ & $\begin{array}{c}\text { Оптимистичный } \\
\text { Optimistic }\end{array}$ \\
\hline & 0,1 & 0,5 & 0,86 \\
\hline$Q_{0}$ & 1,785 & 2,031 & 1,975 \\
\hline$Q_{w}$ & 1,017 & 1,321 & 0,935 \\
\hline$Q_{i n j}$ & 2,634 & 3,246 & 2,763 \\
\hline $\begin{array}{l}\text { KИH } \\
\text { ORF }\end{array}$ & 0,134 & 0,153 & 0,148 \\
\hline
\end{tabular}

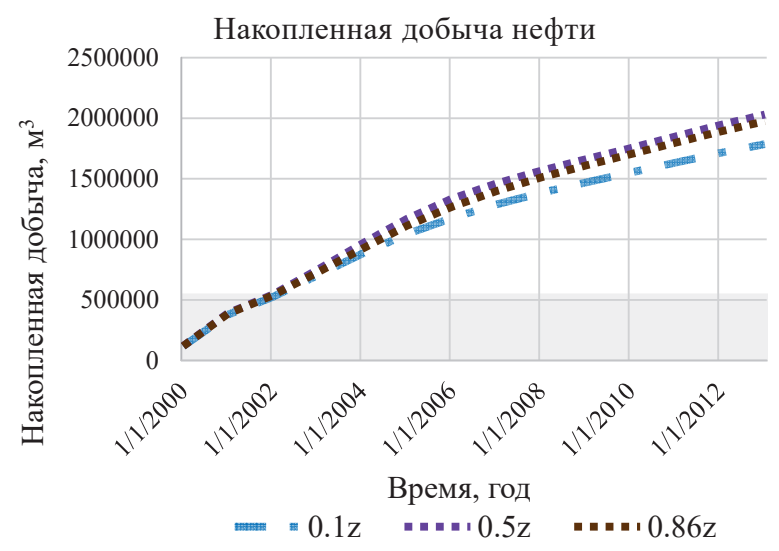

Pис. 3. Сравнение накопленной добычи нефти между тремя вариантами анизотропной модели

Fig. 3. Comparison of cumulative oil production between three variants of anisotropy models

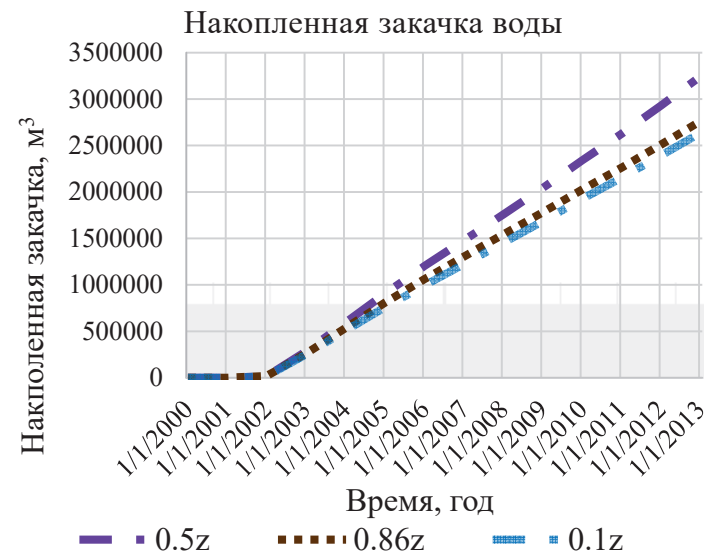

Puс. 4. Сравнение накопленной закачки воды между тремя вариантами анизотропной модели

Fig. 4. Comparison of cumulative water injection between three variants of anisotropy models 
С одной стороны, результаты расчётов имеют схожие тенденции, как и при изменениях значения вертикальной анизотропии, с другой, внедрение латеральной анизотропии в модельный расчёт в общем увеличило значения параметров разработки $\left(Q_{0}, Q_{w}, Q_{i n j}\right)$. Таким образом, результаты гидродинамических расчётов подтверждают, что учёт анизотропии проницаемости является важным, так как позволяет получить более близкое к реальности строение резервуара. В дальнейшем это облегчает процесс адаптации модели под исторические данные разработки и повышает степень уверенности в её результатах при прогнозе.

\section{Оптимизация системы разработки и выводы}

Для оценки эффекта влияния анизотропии проницаемости далее было проведено сравнение поведения коллектора в изотропном и анизотропном случаях. Результаты расчётов представлены в табл. 4 и рис. 5-7.

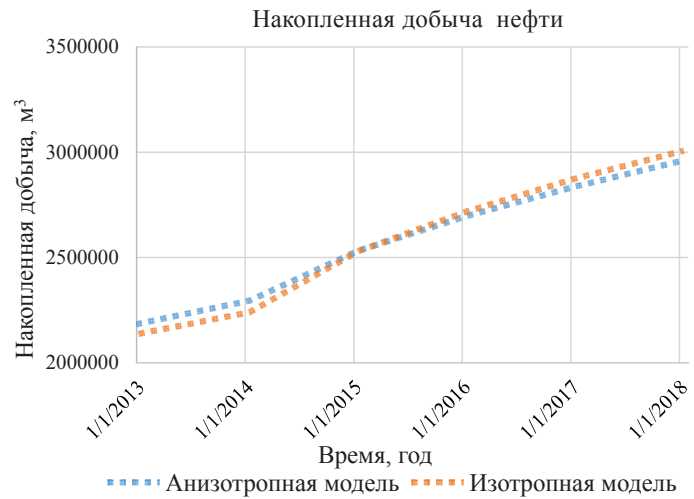

Pис. 5. Сравнение накопленной добычи между изотропной и ани зотропной моделей

Fig. 5. Comparison of cumulative production between isotropy and anisotropy models

Из результатов на рис. 5 видно, что накопленная добыча нефти по изотропной модели оказалась выше накопленной добычи нефти по анизотропной модели. Такой результат указывает на то, что упущение из модельного построения анизотропии может приводить к завышению параметров разработки, что может привести не только к завышению ожидаемой добычи, но и к неправильным экономическим расчётам. Этот факт вновь подтверждает значительное влияние анизотропии проницаемости на процесс моделирования разработки месторождения. В завершение был проведён расчёт на анизотропной модели, учитывающий решения по проведению ГРП в скважинах кандидатах и уплотняющего бурения добывающих и нагнетательных скважин на срок 15 лет (с 2013 г. по 2028 г.). Peзультаты этих расчётов приведены в табл. 4 и рис. 6,7 , где STOIIP - остаточные геологические запасы нефти, (в млн м³), OWC External - внешний контур водонефтяного контакта (ВНК), OWC Internal - внутренний контур BHК, Boundary - границы участка, Oil - нефтедобывающие скважины, In- jection water - водонагнетательные скважины. Важным выводом из результатов является то, что учёт анизотропии проницаемости позволяет определить наиболее вероятное движение флюидов и трещин, создаваемых при проведении ГРП, что подтверждается модельными расчётами [12]. Это видно на рис. 6 , так как начиная с 2013 г. происходит выполнение операций ГРП и резко увеличивается добыча жидкости в добывающих скважинах, находящихся на параллельной оси с нагнетательными скважинами. Уплотняющее бурение добывающих скважин с горизонтальными стволами вдоль оси улучшенных свойств позволило существенно повысить добычу нефти до уровней, близких к начальному периоду разработки участка [13]. Этому также поспособствовало бурение боковых стволов на добывающих скважинах в зонах с ухудшенными свойствами, что привело к увеличению выработки остаточных запасов в таких зонах. Также уплотняющее бурение было выполнено и для нагнетательных скважин. Можно отметить, что в результате этого происходит резкое увеличение добычи жидкости с большим количеством воды и с 2018 г. происходит перекомпенсация пластового давления системой поддержания пластового давления (ППД). Однако, несмотря на данное следствие, это также внесло существенный вклад в эффективность разработки залежи и позволило продолжать рентабельную добычу углеводородов в условиях повышенной обводненности [14-20]. Начиная с 2023 г. произойдет поэтапное отключение части нагнетательных скважин.

таблица 4. Результаты расчётов улучшенной систелы разработки

Table 4. Calculation results of improved development system

\begin{tabular}{|c|c|c|}
\hline \multirow{2}{*}{} & \multicolumn{2}{|c|}{$\begin{array}{c}\text { Параметры разработки, млн }{ }^{3} \\
\text { Development parameters, } \text { MM m}^{3}\end{array}$} \\
\cline { 2 - 3 } & 5 лет (years) & 15 лет (years) \\
\hline$Q_{o}$ & 3,046 & 3,700 \\
\hline$Q_{w}$ & 5,237 & 11,998 \\
\hline$Q_{i n j}$ & 8,745 & 16,607 \\
\hline КИН/ORF & 0,231 & 0,278 \\
\hline
\end{tabular}

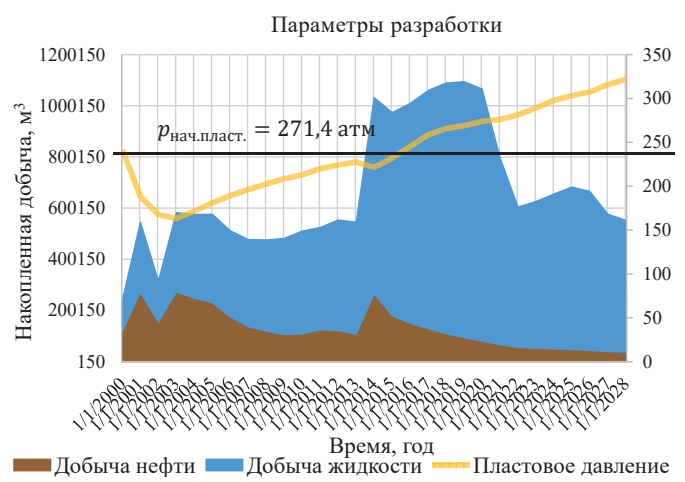

Puс. 6. Накопленная добыча по нефти и жидкости и диналика пластового давления выбранного варианта разработки

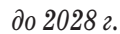

Fig. 6. Accumulation of oil and liquid production and reservoir pressure dynamic of selected development option until 2028 


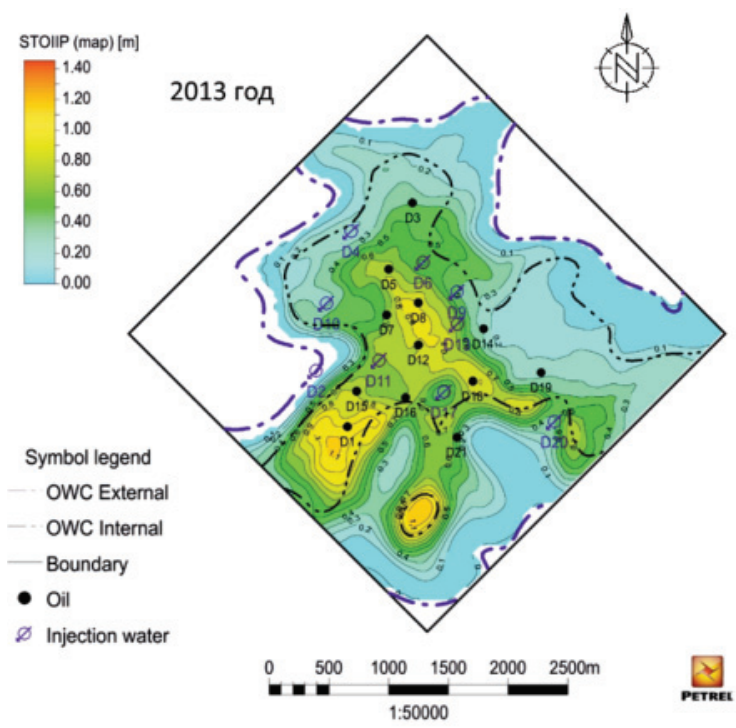

Puc. 7. Карты остаточных запасов

Fig. 7. Maps of remaining oil in place

\section{Заключение}

Таким образом, введение в геолого-гидродинамическую модель величин вертикальной и латеральной анизотропии проницаемости имеет существенное влияние на поведение коллектора. Как показали результаты расчётов, построение изотропной модели может привести к упущению из

\section{СПИСОК ЛИТЕРАТУРЫ}

1. Меркулов В.П., Краснощекова Л.А. Исследование пространственной литолого-петрофизической неоднородности продуктивных коллекторов месторождений нефти и газа // Известия Томского политехнического университета. - 2002. - Т. 305. № 6. - C. 296-303.

2. Two-dimensional flow in a porous medium with general anisotropy Department of Mathematical Sciences and Technology (Norwegian University of Life Sciences). 2004. Available at: http://www.cost734.eu/reports-and-presentations/6th-management-committee-meeting-in-oscarsborg/oscarsborg_p14.pdf (дата обращения 05.08.2018).

3. Краснощекова Л.А., Меркулов В.П. Литолого-петрофизическая анизотропия песчано-глинистых коллекторов нефтяных месторождений // Научно-технический вестник ЮКОС. 2004. - № 10. - С. 33-36.

4. Петрель разработка. Общая информация // Интегрированные программные решения «Шлюмберже». 2018. URL http://sis.slb.ru/products/petrel/petrel_reservoir_engineering (дата обращения 05.08.2018).

5. Amaefule J.O., Altunbay M., Tiab D., Kersey D.G., Keelan D.K. Enhanced reservoir description: using core and log data to identify hydraulic (flow) units and predict permeability in uncored intervals/wells, SPE 26436. $68^{\text {th }}$ Ann. Tech. Conf. and Exhibit. Houston, Tx., 1993. - P. 205-220.

6. Geostatistical conditional simulation // PetroWiki. 2018. URL: https://petrowiki.org/Geostatistical_conditional_simulation (дата обращения: 06.08.2018).

7. Панков М.В. Анализ разработки Крапивинского нефтяного месторождения: отчёт. - Томск: Центр профессиональной переподготовки специалистов нефтегазового дела, 2004. - 425 с.

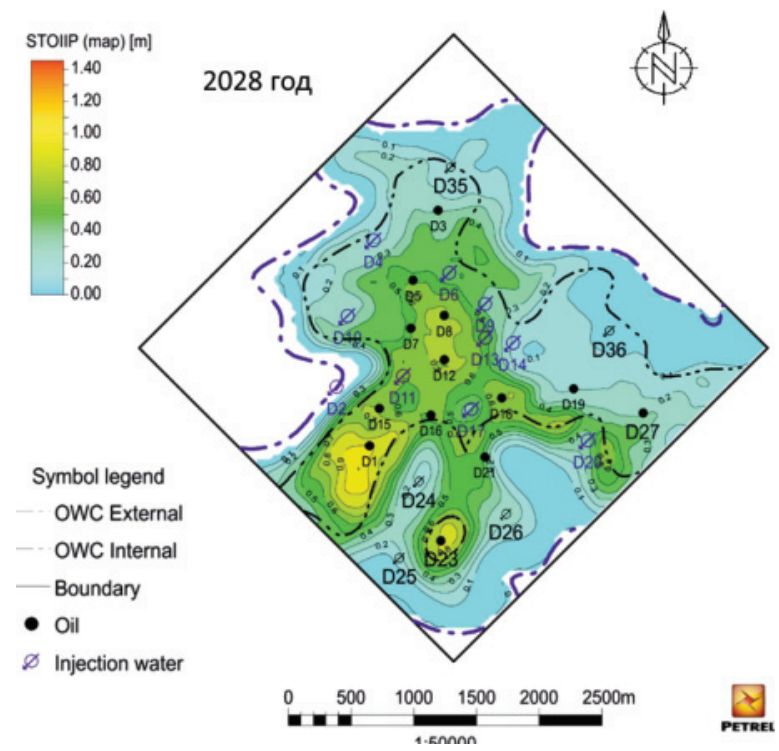

накопленного показателя по добычи нефти до $1 \%$ от того же значения по изотропной модели. Как следствие, при дальнейшем моделировании предлагаемых операций по увеличению нефтеотдачи данное отклонение будет только увеличиваться, что будет создавать искаженную картину разработки.

8. Gray D.H., Fatt I., Bergamini G. The effect of stress on permeability of sandstone cores. - California: Society of Petroleum Engineers, 1963. - V. 3. - P. 95-99.

9. Wannell M.J., Colley N.M., Halford F.R. The use of a new technique to determine permeability anisotropy / Schlumberger Evaluation \& Production Services. - 1993. - V. 12. - P. 489-495.

10. Cosan A. Measuring permeability anisotropy: the latest approach // Oilfield Review. - 1994. - V. 4 - P. 24-35.

11. Коровин М.0. Специализированный анализ керна для изучения анизотропии коллекторов нефти и газа // Геофизика. 2014. - T. 324. - № 1. - C. 87-92.

12. Жабрев И.П., Стуканогов Ю.А. Зависимость нефтеотдачи от направления вытеснения нефти водой из анизотропных по проницаемости пластах // Геология нефти и газа. - 1992. № 8. - C. $34-36$.

13. Al-Hadrami H.K., Teufel L.W. Influence of permeability anisotropy and reservoir heterogeneity on optimization of infill drilling in naturally fractured tight-gas Mesaverde sandstone reservoirs, San Juan Basin // SPE Rocky Mountain Regional: Low permeability reservoirs symposium. - Denver, USA, March 12-15, 2000. - 12 p.

14. Carpenter C. Better permeability estimation from wireline formation testing // Journal of Petroleum Technology. - February, 2018. - V. 70. - P. 66-68.

15. Makhenko R.Y., Tarokh A. Anisotropy in the undrained pore pressure response of rock $/ / 52^{\text {nd }}$ U.S. Rock mechanics/geomechanics symposium. - Seattle, Washington, USA, 17-20 June, 2018. $-7 \mathrm{p}$.

16. Abedi R., Clarke P.L. Modeling of rock inhomogeneity and anisotropy by explicit and implicit representation of microcracks // $52^{\text {nd }}$ U.S. Rock mechanics/geomechanics symposium. - Seattle, Washington, USA, 17-20 June, 2018. - 11 p. 
17. Zhang Z., Du J. Reservoir characterization using perforation shots: anisotropy and attenuation // SEG International Exposition and Annual Meeting. - Anaheim, California, USA, 14-19 October, 2018. $-5 \mathrm{p}$.

18. Kolbikov S., Kuznetsova Y., Smirnov A. Method of anisotropy modeling and its application to hydrodynamic simulation // SPE Russian Petroleum Technology Conference. - Moscow, Russia, 15-17 October, 2018. $-9 \mathrm{p}$.
19. Pan Y., Medhat M.K., Wayne N. Fieldwide determination of directional permeabilities using transient well testing // SPE reservoir evaluation \& engineering. - May, 2019. - V. 22. - 11 p.

20. Liu S., King M.J. Improved calculation of effective permeability for pore network models using the diffuse source methodology: SPE European featured at $81^{\text {st }}$ EAGE Conference and Exhibition. - London, England, UK, 3-6 June, 2019. - 19 p.

Поступила 29.10.2019 2.

\section{Информация об авторах}

Ермеков Р.И., аспирант направления «Геология, разведка и разработка полезных ископаемых» Инженерной школы природных ресурсов Национального исследовательского Томского политехнического университета.

Коровин M. О., кандидат геолого-минералогических наук, инженер лаборатории геологии месторождений нефти и газа Инженерной школы природных ресурсов Национального исследовательского Томского политехнического университета.

Меркулов В.П., кандидат геолого-минералогических наук, ведущий эксперт Центра подготовки и переподготовки специалистов нефтегазового дела Национального исследовательского Томского политехнического университета.

Чернова O.C., доктор геолого-минералогических наук, ведущий эксперт Центра подготовки и переподготовки специалистов нефтегазового дела Национального исследовательского Томского политехнического университета. 


\title{
CONSTRUCTION OF ANISOTROPY SIMULATION MODEL AND STUDYING THE EFFECT OF PERMEABILITY ANISOTROPY ON THE EXAMPLE OF FIELD UNIT MODELING
}

\author{
Roman I. Ermekov', \\ trommka@mail.ru \\ Mikhail O. Korovin', \\ korovinmo@hw.tpu.ru \\ Vitaliy P. Merkulov', \\ merkulovvp@hw.tpu.ru \\ Oksana S. Chernova', \\ merkulovvp@hw.tpu.ru \\ ' National Research Tomsk Polytechnic University, \\ 30, Lenin Avenue, Tomsk, 634050, Russia.
}

The relevance of the research is determined by the fact that the availability of a geological and simulation model of a field adapted to historical data is a powerful tool, which helps engineers control development and consider the effectiveness of the proposed solutions in the near future. Therefore, to obtain reliable results, the quality of constructing reservoir model, taking into account their geological features, is very important. The permeability anisotropy can be considered as one of these models. It is especially important when considering reservoirs with low poroperm properties, the development of which is currently actively started.

The object of the study is one of oil fields of the Tomsk region, consisting of terrigenous sediments. A feature of the field is its location in the system of complex local lifted blocks of various orders. The main productive reservoir is represented by Jurassic sediments, which formed in a regressive-transgressive sedimentation series and having a complex distribution of poroperm properties.

The research methods are based on constructing a field model and conducting simulation calculations, including the selection of permeability anisotropy close to reality, adaptation of an existing development system, as well as construction and analysis of development maps.

The aim of the research includes the assessment of the effect of permeability anisotropy on behavior of reservoir simulation model of oil fields.

The aim of the research. It was found that the omission of permeability anisotropy leads to overestimation of cumulative fluid production of field development. Moreover, an increase of anisotropy magnitude does not always lead to increase in cumulative oil production, which undoubtedly emphasizes the peculiarity of geological structure of reservoir. In conclusion, a simulation calculation of the development was carried out in a period 15 years, the results of which also led to conclusions about correctness of use of operations to increase oil recovery.

Key words:

Permeability anisotropy, anisotropy scale, anisotropy direction, simulation modeling, development strategy.

\section{REFERENCES}

1. Merkulov V.P., Krasnoshchekova L.A. Study of spatial litho-petrophysical heterogeneity of productive collectors of oil and gas deposits. Bulletin of the Tomsk Polytechnic University, 2002, vol. 305 , no. 6, pp. 296-303. In Rus.

2. Two-dimensional flow in a porous medium with general anisotropy Department of Mathematical Sciences and Technology (Norwegian University of Life Sciences). 2004. Available at: http://www.cost734.eu/reports-and-presentations/6th-management-committee-meeting-in-oscarsborg/oscarsborg_p14.pdf (accessed 05 August 2018).

3. Krasnoshchekova L.A., Merkulov V.P. Litologo-petrofizicheskaya anizotropiya peschano-glinistykh kollektorov neftyanykh mestorozhdeniy [Lithology-petrophysical anisotropy of sand-clay collectors of oil fields]. Nauchno-tekhnicheskiy vestnik YUKOS, 2004 , vol. 10 , pp. 33-36.

4. Petrel development.General information. 2018. Integrated software solutions «Gateway». Available at: http://sis.slb.ru/ products/pet$\mathrm{rel} /$ petrel reservoir engineering (accessed 05 August 2018).

5. Amaefule J.0., Altunbay M., Tiab D., Kersey D.G., Keelan D.K. Enhanced reservoir description: using core and log data to identify hydraulic (flow) units and predict permeability in uncored intervals/wells, SPE 26436. $68^{\text {th }}$ Ann. Tech.Conf. and Exhibit. Houston, Tx., 1993. pp. 205-220.
6. Geostatistical conditional simulation. 2018. PetroWiki. Available at: https://petrowiki.org/Geostatistical_conditional_simulation (accessed 06 August 2018).

7. Pankov M.V. Analiz razrabotki Krapivinskogo neftyanogo mestorozhdeniya: otchet [Analysis of development of the Krapivinsky oil field: report]. Tomsk, Center for Professional Retraining of Oil and Gas Specialists, 2004. 425 p.

8. Gray D.H., Fatt I., Bergamini G. The effect of stress on permeability of sandstone cores. California, Society of Petroleum Engineers, 1963. Vol. 3, pp. 95-99.

9. Wannell M.J., Colley N.M., Halford F.R. The use of a new technique to determine permeability anisotropy. Schlumberger Evaluation \& Production Services, 1993, vol. 12, pp. 489-495.

10. Cosan A. Measuring permeability anisotropy: the latest approach. Oilfield Review, 1994, vol. 4, pp. 24-35.

11. Korovin M.O. Specializirovanny analiz kerna dlya izucheniya anizotropii kollektorov nefti i gaza [Specialized analysis of core for study of anisotropy of oil and gas collectors]. Geofizika, 2014, vol. 1, pp. 87-92.

12. Zhabrev I.P., Stukanogov Yu.A. Zavisimost nefteotdachi ot napravleniya vytesneniya nefti vodoy iz anizotropnykh po pronitsaemosti plastakh [Dependence of oil recovery on direction of oil displacement with water from anisotropic by permeability formations]. Geologiya nefti i gaza, 1992, vol. 8, pp. 34-36. 
13. Al-Hadrami H.K., Teufel L.W. Influence of permeability anisotropy and reservoir heterogeneity on optimization of infill drilling in naturally fractured tight-gas Mesaverde sandstone reservoirs, San Juan Basin. SPE Rocky mountain regional: low permeability reservoirs symposium. Denver, USA, March 12-15, 2000. $12 \mathrm{p}$.

14. Carpenter C. Better permeability estimation from wireline formation testing. Journal of Petroleum Technology, February, 2018, vol. 70 , pp. 66-68.

15. Makhenko R.Y., Tarokh A. Anisotropy in the undrained pore pressure response of rock. $52^{\text {nd }}$ U.S. Rock mechanics/geomechanics symposium. Seattle, Washington, USA, 17-20 June, 2018. $7 \mathrm{p}$.

16. Abedi R., Clarke P.L. Modeling of Rock Inhomogeneity and Anisotropy by Explicit and Implicit Representation of Microcracks. $52^{\text {nd }}$ U.S. Rock mechanics/geomechanics symposium. Seattle, Washington, USA, 17-20 June, 2018. 11 p.
17. Zhang Z., Du J. Reservoir characterization using perforation shots: anisotropy and attenuation. SEG International Exposition and Annual Meeting. Anaheim, California, USA, 14-19 0ctober, 2018. 5 p.

18. Kolbikov S., Kuznetsova Y., Smirnov A. Method of Anisotropy Modeling and its Application to Hydrodynamic Simulation. SPE Russian Petroleum Technology Conference. Moscow, Russia, 15-17 October, $2018.9 \mathrm{p}$.

19. Pan Y., Medhat M.K., Wayne N. Fieldwide determination of directional permeabilities using transient well testing. SPE Reservoir Evaluation \& Engineering, May 2019. Vol. 22, 11 p.

20. Liu S., King M.J. Improved calculation of effective permeability for pore network models using the diffuse source methodology. SPE European featured at $81^{\text {st }}$ EAGE Conference and Exhibition. London, England, UK, 3-6 June, 2019. 19 p.

Received: 29 October 2019

\section{Information about the authors}

Roman I. Ermekov, postgraduate, National Research Tomsk Polytechnic University.

Mikhail O. Korovin, Cand. Sc., engineer, National Research Tomsk Polytechnic University.

Vitaliy P. Merkulov, Cand. Sc., leading expert, National Research Tomsk Polytechnic University.

Oksana S. Chernova, Dr. Sc., leading expert, National Research Tomsk Polytechnic University. 